are uncertain whether it is part of their role at all. However the NICE Supportive \& Palliative Care Improving Outcomes Guidance 2004, in dedicating a whole chapter to this topic, made it clear that this part of holistic assessment and care falls squarely within the remit of healthcare staff. Subsequent national documents confirm this.

Aims To assess the impact on frontline clinical staff, of face to face (F2F) and online versions, of a course designed to raise spiritual awareness.

Method A qualitative study involving thematic analysis of semi structured, mainly open question, interviews, offered to the last 100 each of online and F2F course attendees. Interviews were offered to $54(27 \%)$ respondents and 24 agreed a mutually convenient time. This part of the evaluation reports the analysis of the interviews with 20 frontline clinicians (4 chaplain interviews form part of another study).

Results Spiritual awareness was achieved independently of the mode of course delivery, although participants articulated preferences. A diverse participant group enjoyed varied learning methods, resources and discussion. Good IT support and lack of technical hitches was important to smooth running online. Positive use of grouping, skilled facilitation (crucial) and the flexibility and anonymity of online learning suits a mixture of clinicians regardless of role and hierarchy. Confidence and structure in conversations with patients, empowering patients to seek innate solutions and strategies for dealing with questions about personal beliefs helped clinicians to address spiritual needs.

Conclusions ' $\mathrm{e}$ '=experiential regardless of mode of delivery. Enthusiastic, more confident, participants recognised significant barriers, requiring organisational recognition for training to address spiritual needs and provide compassionate care.

\section{P 045 WHAT'S THE 'E' IN E-LEARNING FOR SPIRITUALITY: A COMPARISON OF ONLINE AND FACE TO FACE COURSE VERSIONS FOR HEALTHCARE WORKERS}

\author{
Karen Groves, ${ }^{1,2}$ Cath Baldry, ${ }^{1}$ Cathy Sherrat ${ }^{3} .{ }^{1}$ Queenscourt Hospice, Southport, UK; \\ ${ }^{2}$ Southport \& Ormskirk Hospitals' NHS Trust, Southport UK; ${ }^{3}$ Edge Hill University, \\ Ormskirk Rd, Ormskirk, Lancashire UK
}

10.1136/bmjspcare-2014-000654.86

Background Healthcare staff feel unprepared for dealing with spiritual and religious needs of patients and families. Indeed many 